\title{
Reduced Immune Response to Polymeric Micelles Coating Sialic Acids
}

Masashi Ohmae ${ }^{\mathrm{a} *}$, Mei Kojima ${ }^{\mathrm{a}}$, Kenta Mihara ${ }^{\mathrm{a}}$, Yuji Yamazaki ${ }^{\mathrm{a}}$, Isao Hara ${ }^{\mathrm{b}}$, Eri Hara ${ }^{\mathrm{c}}$, and Shunsaku Kimura ${ }^{\mathrm{a}}$

${ }^{a}$ Department of Material Chemistry, Graduate School of Engineering, Kyoto University, Kyoto daigaku-katsura, Nishikyo-ku, Kyoto 615-8510, Japan

${ }^{b}$ Technology Research Laboratory, Shimadzu Corporation, 3-9-4 Hikari-dai, Seika-cho, Soraku-gun, Kyoto 619-0237, Japan

${ }^{c}$ Department of Experimental Therapeutics, Institute for Advancement of Clinical and Translational Science, Kyoto University Hospital, 53 Shogoin kawahara-cho, Sakyo-ku, Kyoto 606-8507, Japan

\footnotetext{
*Corresponding author

Tel.: +81-75-383-2404; fax: +81-75-383-2401

E-mail address: ohmae@peptide.polym.kyoto-u.ac.jp
}

\begin{abstract}
Effects of sialic acid coatings on polymeric micelle consisting of poly(sarcosine)-block-poly(L-lactic acid) (Lactosome) in the aim of prevention of the accelerated blood clearance (ABC) phenomenon are studied. Two kinds of the sialic acid-presenting Lactosomes targeting the immunosuppressive receptors of Siglec-G and CD22 have been successfully prepared. Lactosome presenting 5-N-acetylneuraminic acid or
\end{abstract} 5 - $N$-acetylneuraminyl- $\alpha(2 \rightarrow 6)$-galactosyl- $\beta(1 \rightarrow 4)-N$-acetylglucosamine at the nanocarrier surface 1 
diminished the $\mathrm{ABC}$ phenomenon due to the reduction of the anti-poly(sarcosine) IgM production. Further, the sialic acid moieties could interact possibly with Siglec-E on immune cell to suppress phagocytosis of the opsonized nanocarriers.

Keywords: Lactosome; Accelerated blood clearance phenomenon; Siglecs; Sialic acid; Nanocarriers 
Nanocarriers are currently explored extensively as a vehicle of various medicinal agents in biomedical fields including targeted drug delivery systems (T-DDS), medical imaging, and diagnosis. ${ }^{1,2}$ Particularly in regard of cancer, nanocarriers are believed to become one of the most powerful tools to challenge this tricky disease. ${ }^{3}$ Current cancer treatments include chemotherapy by anticancer agents, radiation therapy, surgical removal of tumor tissues, and in most cases, combination of these treatments and the immune checkpoint antibodies under some circumstances. However, these treatments often cause serious damages to healthy cells in the patients. In order to avoid such side effects, it is desirable to develop cancerous cells-specific T-DDS. Nanocarriers are highly potential to transport a variety of substances with their loading in nanocarriers. Particularly in cancer therapies, nanocarriers can be accumulated in tumor sites due to the characteristic features around the tumor region, i.e., the enhanced permeability and retention (EPR) effect that is explained by extravasation through leaky vessels and poor lymphatic drainage in tumors. ${ }^{4}$ Furthermore, the long-circulating ability (the "stealth" property) of nanocarriers also assists feasibility for the cancer therapies, which can be attainable by surface modification of nanocarriers with the highly hydrated and dense polymers such as poly(ethyleneglycol) $\quad$ (PEG), ${ }^{5}$ poly[N-(2-hydroxypropyl)methacrylamide] (HPMA), ${ }^{6}$ poly(hydroxyethyl-L-asparagine) (PHEA), ${ }^{7}$ chitosan, ${ }^{8}$ etc. Normally, the intravenously administered nanocarriers without the stealth functionalization are rapidly cleared by the mononuclear phagocyte system (MPS) within several minutes. Thus the stealth nanocarriers are the first choice in DDS.

The stealth nanocarriers, however, have been pointed out to lose the stealth property upon multiple administrations. For example, the stealth PEGylated liposomes become "visible" for the MPS at the second administration and after that. This change has been known as the accelerated blood clearance (ABC) phenomenon. ${ }^{9,10}$ The ABC phenomenon was also observed with the PEGylated nanocarrier, which was found to be recognized by B lymphocyte through the PEG chains as the T cell-independent type 2 (TI-2) antigen, ${ }^{11}$ leading to production of anti-PEG IgM. ${ }^{12,13}$ Many 
efforts have been made in order to avoid the $\mathrm{ABC}$ phenomenon, but it is still challenging to date. ${ }^{14}$

Previously, we have established the novel stealth nanocarrier consisting of amphiphilic block copolymers of poly(sarcosine)-block-poly(L-lactic acid) (PSar-PLLA, 1) named as "Lactosome" (Fig. 1a-b). ${ }^{15}$ Poly(sarcosine) is a hydrophilic polypeptide, which provides the hydrophobic core of poly(L-lactic acid) in Lactosome with the sterically hindered hydrophilic shell. Lactosome is very effective as a vehicle for delivery to various solid tumors. ${ }^{16,17}$ However, the ABC phenomenon was observed similarly to the PEGylated liposome. ${ }^{18}$ The intensive studies revealed that the ABC phenomenon induced by Lactosome was due to the production of anti-poly(sarcosine) $\operatorname{IgM}$ and $\operatorname{IgG}_{3}$ by the direct activation of the peritoneal B lymphocyte. ${ }^{19-21}$ The ABC phenomenon has been shown to be solved out by using $\mathrm{A}_{3} \mathrm{~B}$-type $\left(((\text { poly(sarcosine }))_{3}\right.$-block-poly(L-lactic acid)) polymer ${ }^{22}$ or taking a nano-sheet morphology, ${ }^{23}$ which are based on the concept to increase the surface density of poly(sarcosine) chains on the nanocarrier surface to prevent the B lymphocyte recognition. On the other hand, it has been pointed out that the activation of the B cell subset can be inhibited by the suppressive ligand of sialic acid concurrently associating with Siglec of the B cell. ${ }^{24}$ Siglecs are the family of sialic acid-binding immunoglobulin-like lectins found mainly on immune cells, which are believed to regulate a variety of immune cell functions. Siglec-G and CD22 on mouse B cells have been reported as the critical Siglecs responsible for suppression of the immune response to TI-2 antigens via induction of apoptosis. ${ }^{25-27}$ We thus report here the availability of the surface modification of Lactosome with sialic acid to escape from the $\mathrm{ABC}$ phenomenon. 
(a)

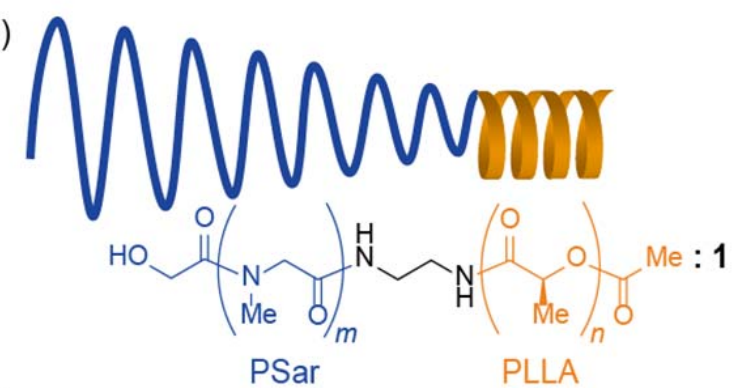

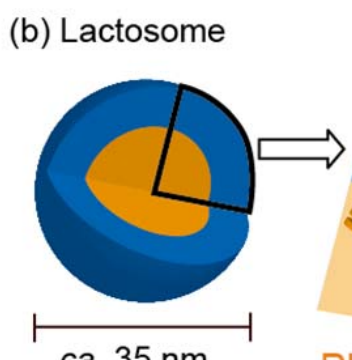

ca. $35 \mathrm{~nm}$

(c) $\mathrm{OH}$

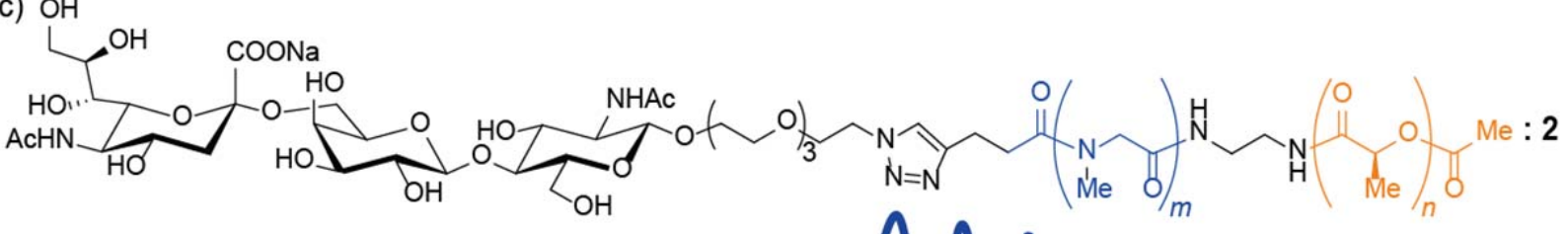

(d) $\mathrm{OH}$
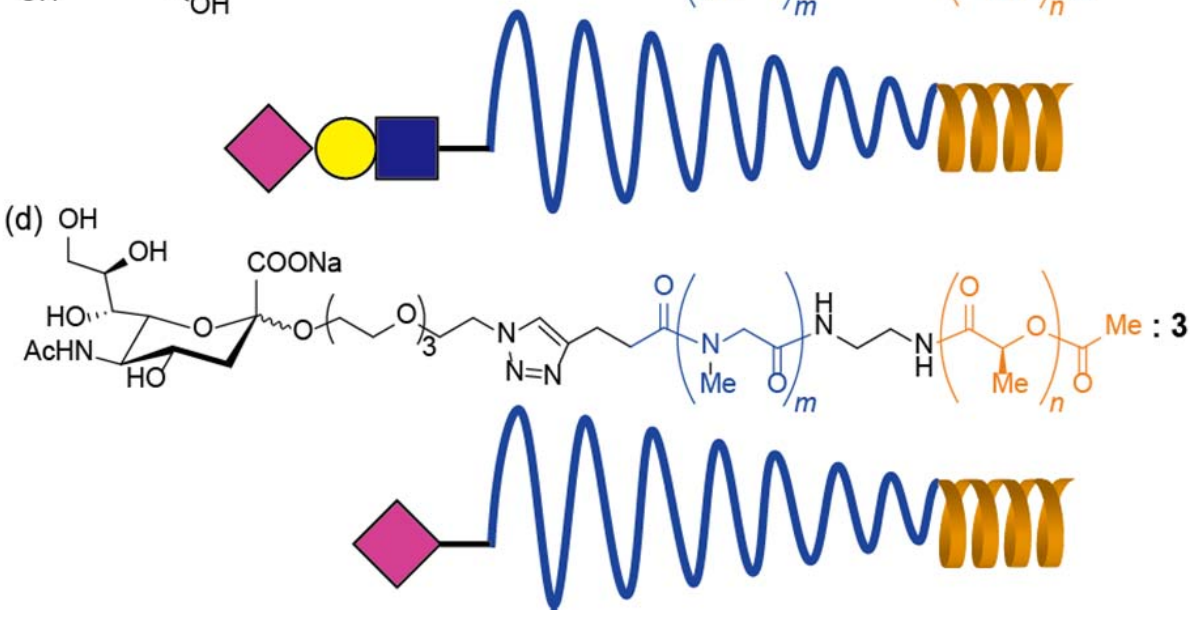

Figure 1. Schematic illustrations of Lactosome and the glycan-coated Lactosomes: (a) chemical structure of the component block copolymer PSar-PLLA, (b) formulation of Lactosome, (c) the PSar-PLLA with a Neu5Ac $\alpha(2 \rightarrow 6) \mathrm{Gal} \beta(1 \rightarrow 4)$ GlcNAc $\beta$ - at the terminal (2), (d) the PSar-PLLA with a Neu5Ac at the terminal (3).

The ligands for Siglec-G and CD22 have been reported to be the sialoglycans, i.e., those having $\operatorname{Sia} \alpha(2 \rightarrow 6) \mathrm{Gal} \beta(1 \rightarrow 4)$ GlcNAc $\beta \quad$ terminal structures, where Sia represents a $5-N$-glycolylneuraminic acid $(\mathrm{Neu} 5 \mathrm{Gc})$ or a 5 - $N$-acetylneuraminic acid (Neu5Ac) in mice. ${ }^{24}$ The latter is a common carbohydrate structure in both humans and mice. We therefore chose $\operatorname{Neu} 5 \operatorname{Ac} \alpha(2 \rightarrow 6) \operatorname{Gal} \beta(1 \rightarrow 4)$ GlcNAc $\beta$ connecting to the terminal of the poly(sarcosine) chains (2) (Fig. 1c) to construct the glycan-coated Lactosome. In order to evaluate the effect of sialic 
acid-coatings, ${ }^{28,29}$ the monosaccharidic Neu5Ac ligand at the poly(sarcosine) terminal (3) (Fig. 1d) was also prepared and studied. Retrosynthetic analysis of 2 is shown in Scheme 1. Copolymer 2 should be formed by ligation of the terminal azido functionalized carbohydrate part of 4 and the amphiphilic block copolymer part of 5 bearing an alkyne moiety via the Huisgen cycloaddition. Copolymer 5 should be prepared by condensation of pentynoic acid 6 and the copolymer $7 .^{15}$ The carbohydrate part of $\mathbf{4}$ is formed by glycosylation of the sialic acid donor $\mathbf{8}^{30}$ to the novel disaccharide derivative 9 followed by deprotections. The derivative 9 is obtained by glycosylation of the glycosyl donor $\mathbf{1 0}^{31}$ to the commercially available azido functionalized oligoethyleneoxide alcohol 11
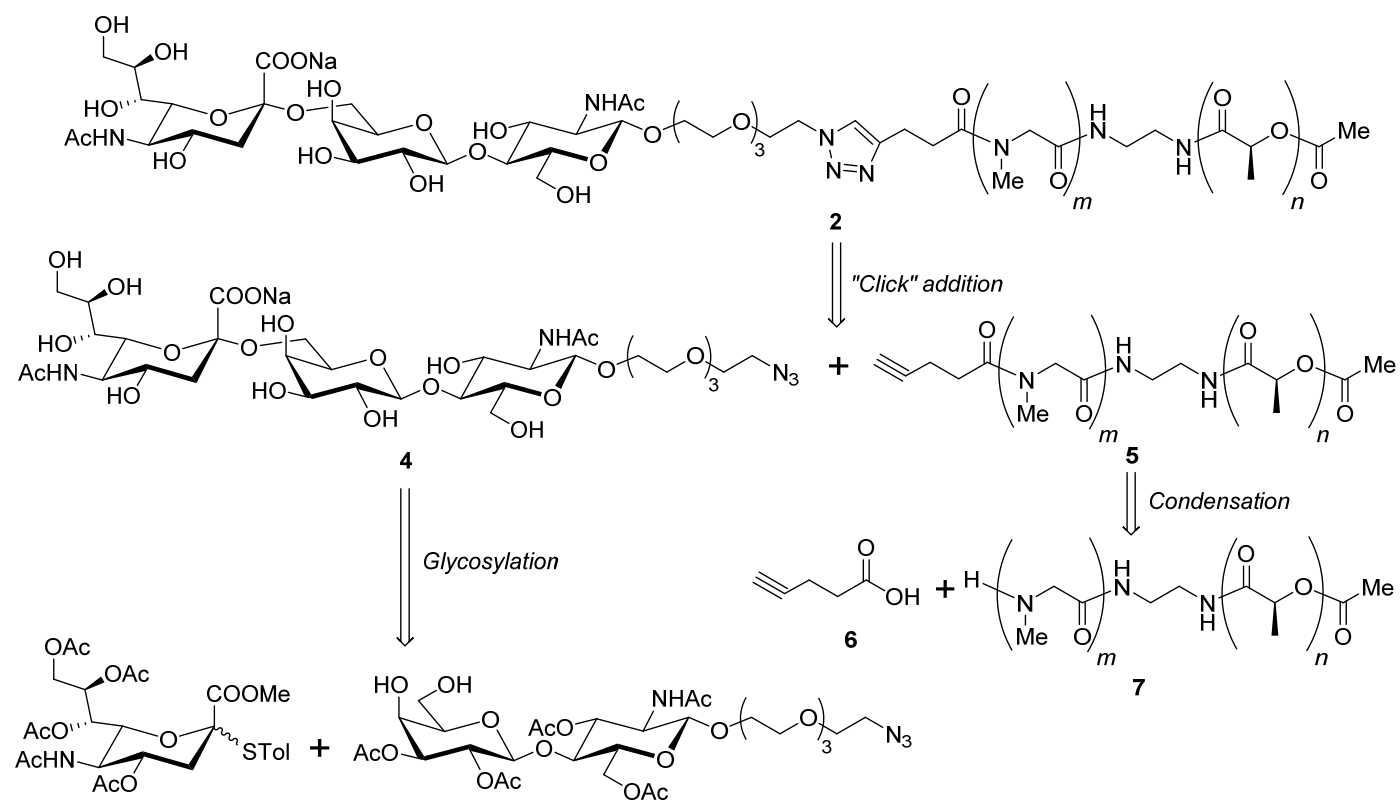

8
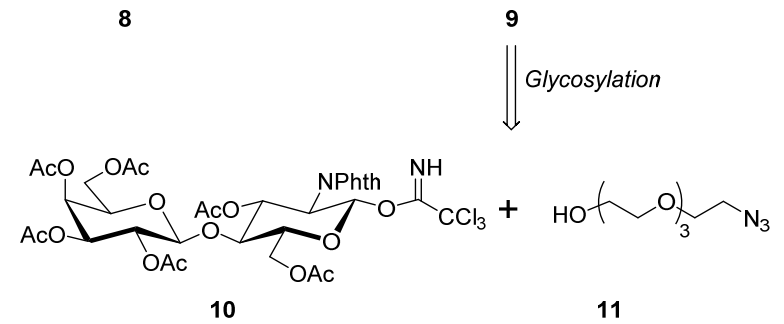

Scheme 1. Retrosynthetic analysis of 2 
Copolymer 2 was synthesized according to the reactions outlined in Scheme 2. Compound 11 was successfully glycosylated with $\mathbf{1 0}$ to afford $\mathbf{1 2}$ in a 90\% yield. All of the protecting groups in $\mathbf{1 2}$ were removed, then peracetylation was carried out to afford 13. O-Acetyl protection in $\mathbf{1 3}$ was fully removed to obtain 14 in a $95 \%$ yield, and then it was treated with 4-anisaldehyde dimethylacetal and catalytic amount of 10-camphorsulfonic acid, followed by acetylation and removal of 4',6'-(4-methoxybenzylidene) group to provide 9 in a $88 \%$ yield via 3 steps. Regioselective $O$-sialylation of $\mathbf{9}$ was carried out with $\mathbf{8}$ under the conditions described in "Supplementary data" section. The products were obtained as the mixture of the diastereomers $(\alpha / \beta=3 / 1)$ in a $70 \%$ yield. Fortunately the target compound of 15 ( $\alpha$-anomer) was separated by silica gel column chromatography in a $43 \%$ yield. All of the $O$-acetyl groups and the methyl ester in $\mathbf{1 5}$ were removed successively by sodium methoxide in methanol and then by $1 \mathrm{M}$ aq $\mathrm{NaOH}$ at $0{ }^{\circ} \mathrm{C}$, to give 4 in a 74\% yield. The terminal alkyne functionalized copolymer 5 was prepared by condensation of pentynoic acid 6 and the amphiphilic block copolymer $7^{15}(m=29, n=30$; as average numbers of monomers determined by ${ }^{1} \mathrm{H}$ NMR measurements) by using 1-[(1-(cyano-2-ethoxy-2-oxoethylideneaminooxy)-dimethylamino-morpholinomethylene)]methanam inium hexafluorophosphate $(\mathrm{COMU})^{32}$ as a coupling reagent. This condensation reaction proceeded almost quantitatively, which was inferred by MALDI-TOF MS. Compound $\mathbf{4}$ and copolymer $\mathbf{5}$ were connected by the Huisgen cycloaddition reaction to afford 2 quantitatively. 

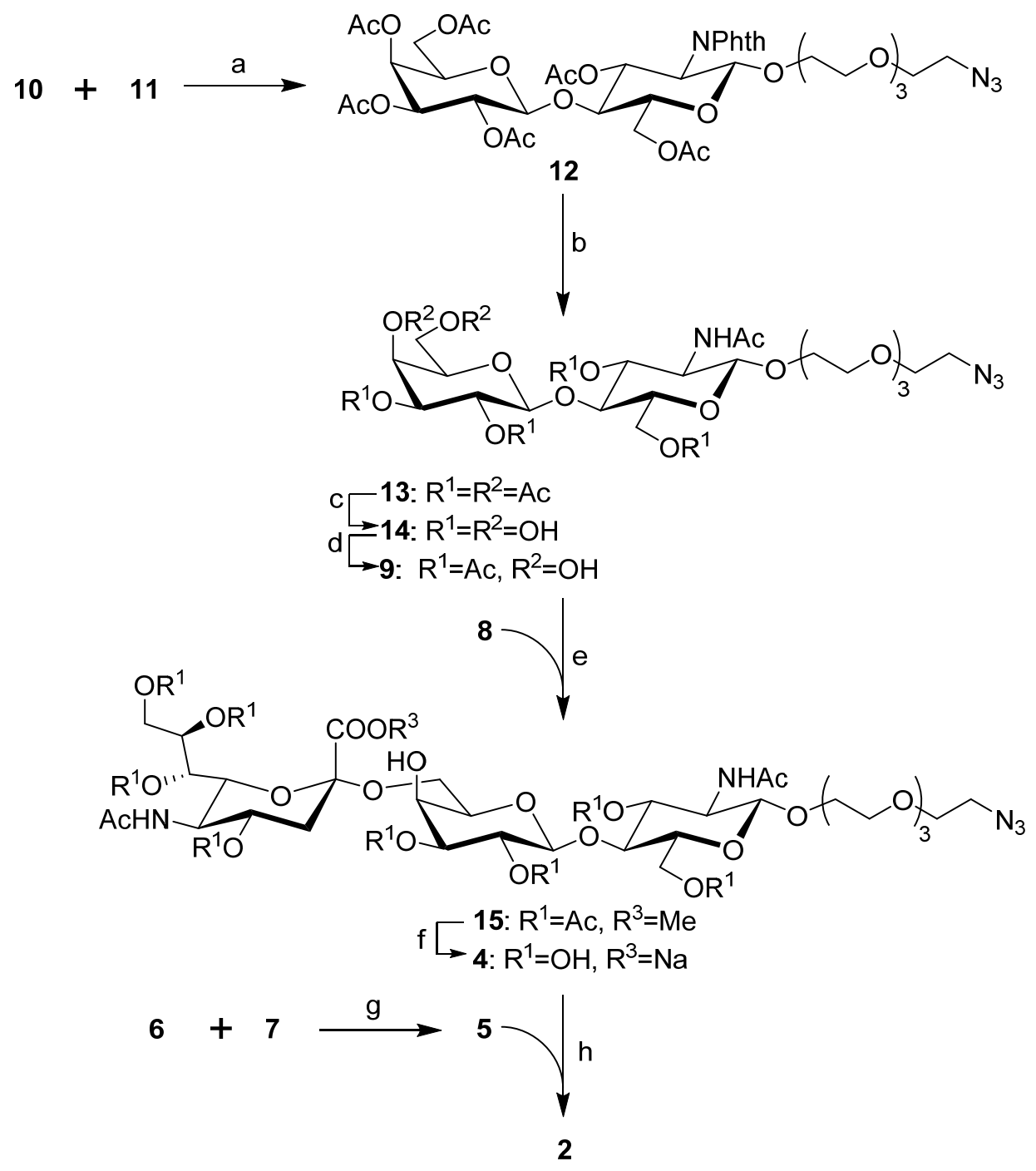

Scheme 2. Reagents and conditions: a) TMSOTf, MS4A / $\mathrm{CH}_{2} \mathrm{Cl}_{2},-20{ }^{\circ} \mathrm{C}, 0.5 \mathrm{~h}, 90 \%$, b) 1) $\mathrm{MeONa} / \mathrm{MeOH}, \mathrm{rt}, 4 \mathrm{~h}, 2) \mathrm{NH}_{2} \mathrm{NH}_{2} \cdot \mathrm{H}_{2} \mathrm{O} / \mathrm{EtOH}$, reflux, 5 h, 3) $\mathrm{Ac}_{2} \mathrm{O} /$ pyridine, rt, overnight, $87 \%$ (3 steps), c) $\mathrm{MeONa} / \mathrm{MeOH}, \mathrm{rt}, 2$ h, 95\%, d) 1) p-anisaldehyde dimethylacetal, CSA / DMF, rt, 2 h, then $\left.30{ }^{\circ} \mathrm{C}, 26 \mathrm{~h}, 2\right) \mathrm{Ac}_{2} \mathrm{O} /$ pyridine, rt, $\left.18 \mathrm{~h}, 3\right) 80 \%$ aq $\mathrm{AcOH}, 50{ }^{\circ} \mathrm{C}, 3 \mathrm{~h}, 88 \%$ (3 steps), e) NIS, $\mathrm{TfOH}, \mathrm{MS} 3 \mathrm{~A} / \mathrm{CH}_{3} \mathrm{CN},-40{ }^{\circ} \mathrm{C}, 72 \mathrm{~h}, 43 \%$ for $\alpha$-anomer, f) 1) $\left.\mathrm{MeONa} / \mathrm{MeOH}, 0{ }^{\circ} \mathrm{C}, 6 \mathrm{~h}, 2\right) 0.2 \mathrm{M}$ aq $\mathrm{NaOH}, 0{ }^{\circ} \mathrm{C}, 4 \mathrm{~h}, 74 \%$ (2 steps), g) COMU, Oxyma pure, DIEA / DMF, $0{ }^{\circ} \mathrm{C}$ then $\mathrm{rt}$, overnight, quant, h) $\mathrm{Cu}(\mathrm{I}) \mathrm{OAc} / \mathrm{CH}_{2} \mathrm{Cl}_{2}-\mathrm{MeOH}, \mathrm{rt}, 15 \mathrm{~h}$, quant.

Copolymer 3 was also prepared chemically according to the reactions outlined in Scheme 3. Compound 11 was glycosylated with 8 under the similar reaction conditions to that for 15, which 
gave 16 as the mixture of $\alpha$ - and $\beta$-anomers $(\alpha / \beta=2 / 1)$ in a $90 \%$ yield. Unfortunately, these diastereomers were difficult to separate by the conventional silica gel column chromatography, leading to the usage of $\mathbf{1 6}$ as an anomeric mixture. All of the $O$-acetyl groups and the methyl ester in 16 were removed to give 17. Compound $\mathbf{1 7}$ and copolymer 5 were also ligated by the Huisgen cycloaddition reaction to give 3 in a quantitative yield.

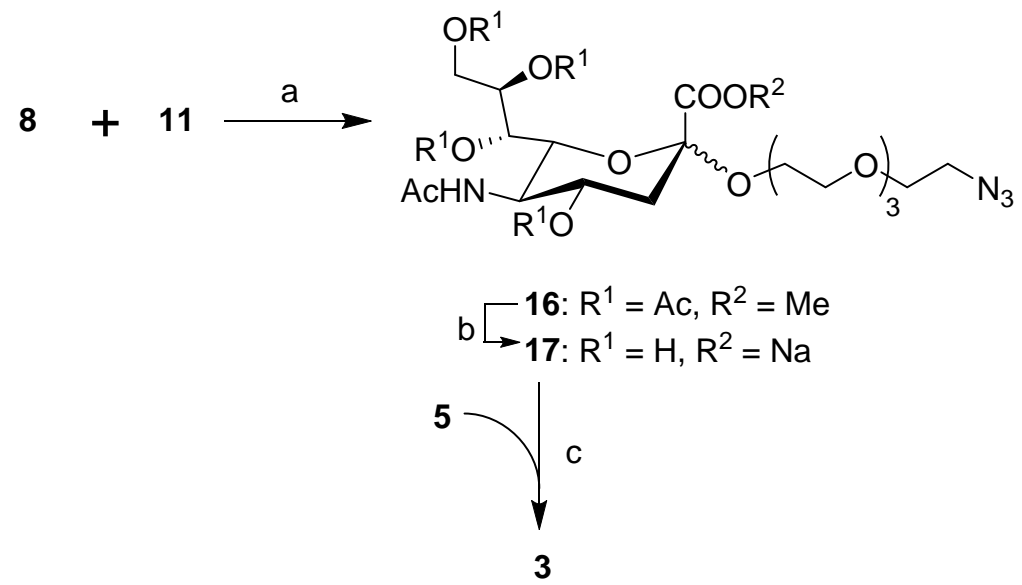

Scheme 3. Reagents and conditions: a) NIS, TfOH, MS3A / $\mathrm{CH}_{3} \mathrm{CN},-40{ }^{\circ} \mathrm{C}, 27 \mathrm{~h}, 90 \%(\alpha / \beta=2 / 1)$, b) 1) $\left.\mathrm{MeONa} / \mathrm{MeOH}, 0{ }^{\circ} \mathrm{C}, 3 \mathrm{~h}, 2\right) 0.1 \mathrm{M}$ aq NaOH$, 0{ }^{\circ} \mathrm{C}, 4 \mathrm{~h}$, quant, c) $\mathrm{Cu}(\mathrm{I}) \mathrm{OAc} / \mathrm{CH}_{2} \mathrm{Cl}_{2}-\mathrm{MeOH}$, rt, $15 \mathrm{~h}$, quant.

Structures of $\mathbf{2}$ and $\mathbf{3}$ were confirmed by MALDI-TOF MS analysis (Fig. 2). The spectrum of $\mathbf{2}$ showed molecular mass distribution ranging from ca. $4.7 \times 10^{3}$ to $6.3 \times 10^{3}$ (Fig. 2a); The mass difference between the peaks was $c a$. 71, which corresponds to the repeating units of sarcosine (71.04) and lactate (72.02) residues. The peak with the highest intensity was found at $\mathrm{m} / \mathrm{z}=5494.281$, which corresponds to the molecular mass of $2(m=27, n=35)$ as a $[\mathrm{M}-\mathrm{Na}]^{-}$form. Similarly, the spectrum of 3 showed the peaks having molecular mass distribution in the range from $c a .4 .4 \times 10^{3}$ to $6.0 \times 10^{3}$. The mass difference between the peaks was also ca. 71 (Fig. 2b). The peak with the highest intensity was found to be $m / z=5273.971$, which agrees with the molecular mass of 3 ( $m=27$, $n=37)$ as a $[\mathrm{M}-\mathrm{Na}]^{-}$form. From the spectrum of 5 (Fig. 2c), the terminal alkyne functionalized 
polymer has molecular mass distribution in the range from ca. $3.7 \times 10^{3}$ to $5.2 \times 10^{3}$. The mass difference between the peaks was also $c a$. 71. The highest peak intensity was observed at $\mathrm{m} / \mathrm{z}=$ 4512.397, which corresponds to the molecular mass of $5(m=30, n=30)$ as a $[\mathrm{M}+\mathrm{K}]^{+}$form. In either spectrum, the conjugation of the sialic acid moiety to the amphiphilic polymer was confirmed.

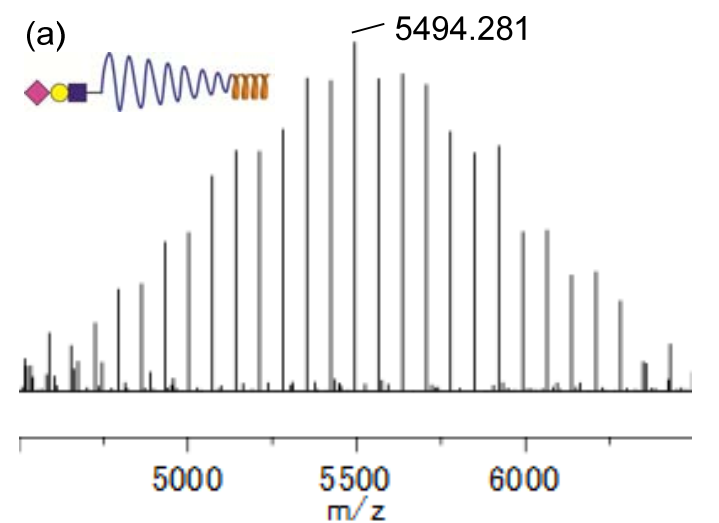

(b)

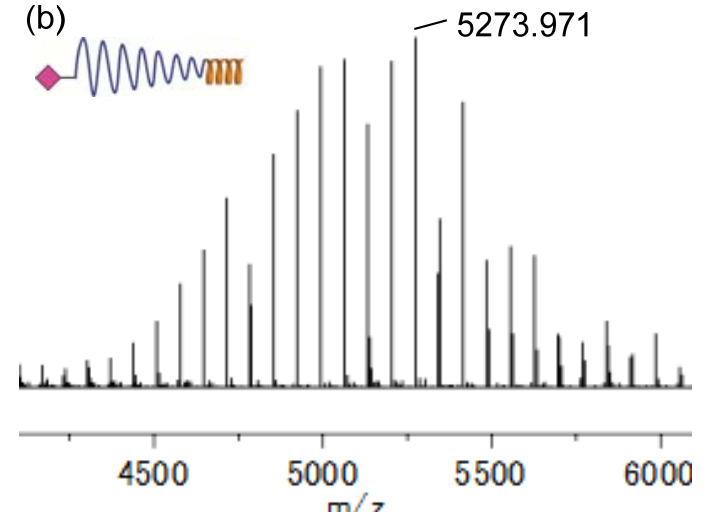

(c) $\quad-4512.397$

MWDinir

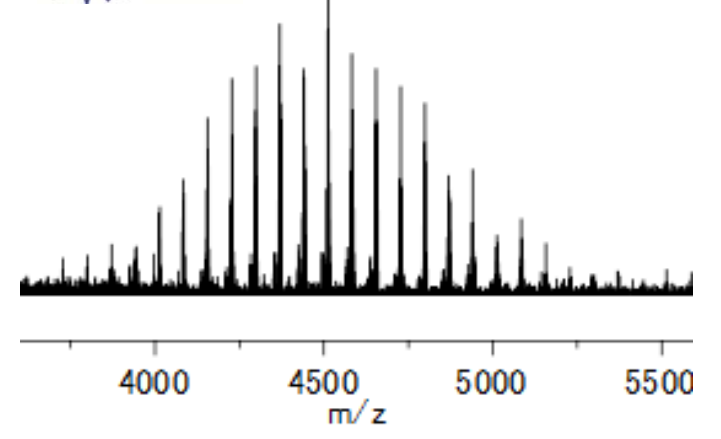

Figure 2. MALDI-TOF mass spectra of (a) 2, (b) 3 and (c) 5. The spectra of 2 and 3 were recorded under the negative ion mode, and that of 5 under the positive ion mode.

Two kinds of the sialic acid-presenting Lactosomes were prepared according to the procedure 
reported previously ${ }^{15}$ with mixing 2 or 3 and PSar-PLLA block copolymer $(m=73, n=30)$ at the molar ratio of 1:1 (Tri-Lacto, containing 2; Mono-Lacto, containing 3). The polymeric micelle composed of PSar-PLLA block copolymer without any mixing of $\mathbf{2}$ or $\mathbf{3}$ was also examined (Lacto). For pharmacokinetic analysis, Lactosomes were labeled with indocyanine green-connected poly(D-lactic acid) (ICG-PDLA) $)^{33} ; 2$ nmol ICG-PDLA per $1 \mathrm{mg}$ of the Lactosomes composed of PSar-PLLA (ICG-Lacto), of PSar-PLLA +2 (ICG-Tri-Lacto), and of PSar-PLLA +3 (ICG-Mono-Lacto). The hydrodynamic diameters were measured by DLS measurements (Table 1) to be in the range of $40-50 \mathrm{~nm} .^{15}$

Table 1. The hydrodynamic diameters of Lactosomes

\begin{tabular}{cccc}
\hline Entry & Lactosome & Diameter $/ \mathrm{nm}$ & Polydispersity index \\
\hline 1 & Tri-Lacto & 44 & 0.08 \\
2 & ICG-Tri-Lacto & 48 & 0.11 \\
3 & Mono-Lacto & 44 & 0.14 \\
4 & ICG-Mono-Lacto & 46 & 0.07 \\
5 & ICG-Lacto & 41 & 0.01 \\
\hline
\end{tabular}

In vivo dispositions of Tri-Lacto and Mono-Lacto in BALB/c nu/nu mice upon multiple administrations were studied by the near-infrared fluorescence (NIRF) imaging method (Fig 3). Lactosome was found to be T-cell independent antigen, and this thymus-lacking immunodeficient mouse is used here for the usefulness to study the pharmacokinetic change by NIRF-imaging. ${ }^{18}$ Accumulation of each nanocarrier at liver was observed at $5 \mathrm{~min}$ and $24 \mathrm{~h}$ after the second administration with different intensities depending on the nanocarrier compositions. It is shown that the intensities at liver (liver accumulation) in the cases of Tri-Lacto and Mono-Lacto at the first administration (Fig. 3 b) j)) are weakened compared with the Lacto administration (Fig. 3 f), k)), 
which suggest some escape ability from the immune system. The time-course of the intensity ratio of liver and background in each mouse was further investigated (Fig. 4). As estimated in the NIRF imaging, the ratio of region of interest (ROI) at liver against background, which is an index for the $\mathrm{ABC}$ phenomenon, increased in all cases but in different degrees. The liver/background ratios at $8 \mathrm{~h}$ after the second administration are compared to show a significant decline of Mono-Lacto/ICG-Mono-Lacto (first administration/second administration), Tri-Lacto/ICG-Lacto, and Tri-Lacto/ICG-Tri-Lacto from Lacto/ICG-Lacto and Mono-Lacto/ICG-Lacto. Similar time-courses between Lacto/ICG-Lacto and Mono-Lacto/ICG-Lacto indicate that Mono-Lacto at the first administration should induced the immune response of the anti-poly(sarcosine) IgM production as effective as Lactosome to induce the ABC phenomenon. Interestingly, ICG-Mono-Lacto at the second administration escaped partially from liver capture despite the activated immune system, which is discussed in the later section. The relatively low ratios of liver against background in the cases of Tri-Lacto/ICG-Lacto and Tri-Lacto/ICG-Tri-Lacto suggest that the Sia trisaccharide decoration suppressed liver uptake of the nanocarriers due to the suppressive production of anti-poly(sarcosine) IgM. 
(a)

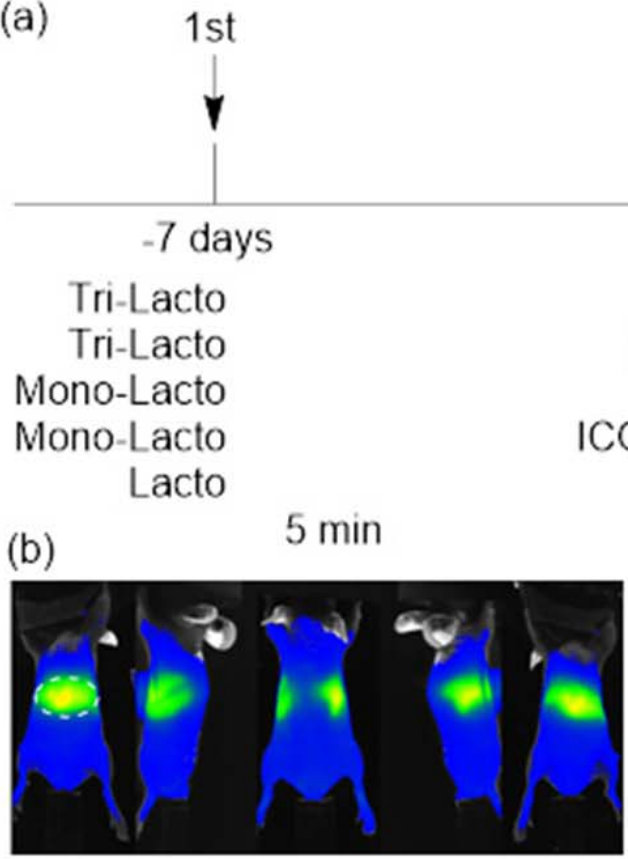

(c)

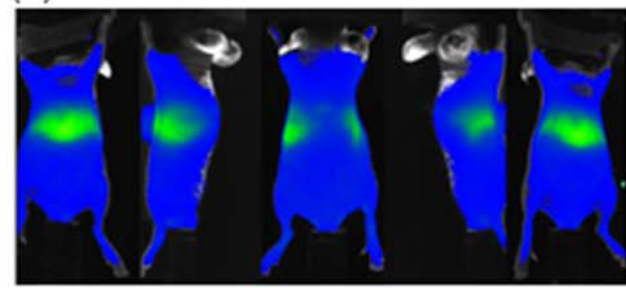

(d)

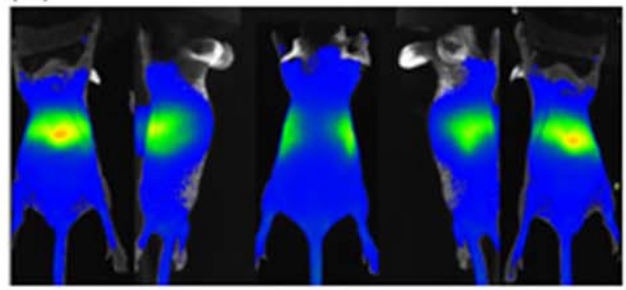

(e)

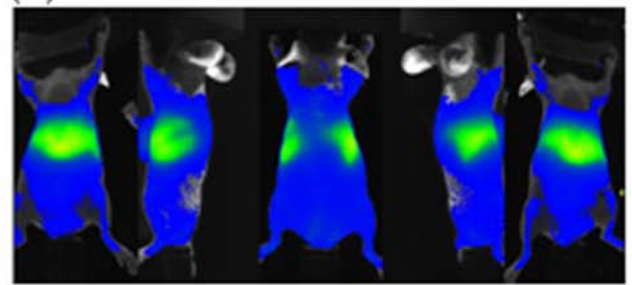

(f)

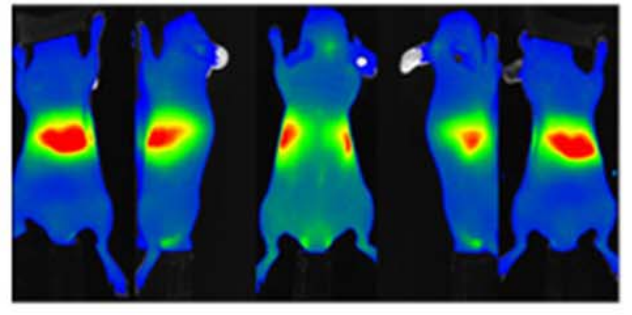

2nd NIRF

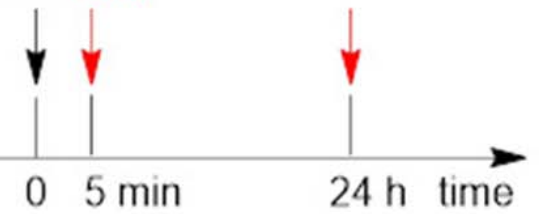

ICG-Lacto (b)

ICG-Tri-Lacto (c)

ICG-Lacto (d)

(g)

(h)

(i)

(j)

(k) (g)

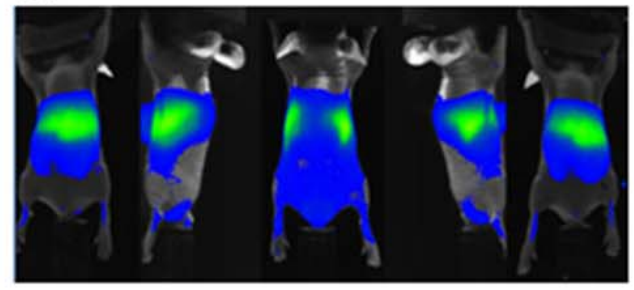

(h)

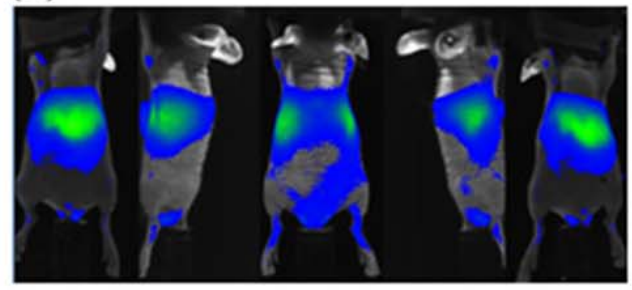

(i)

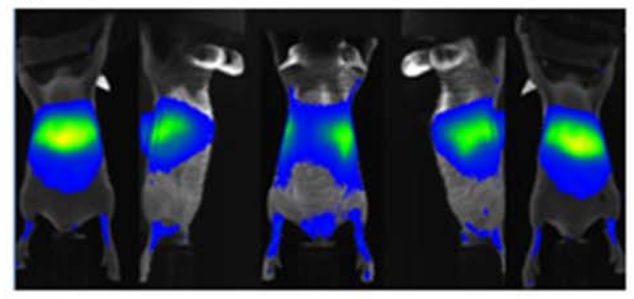

(j)

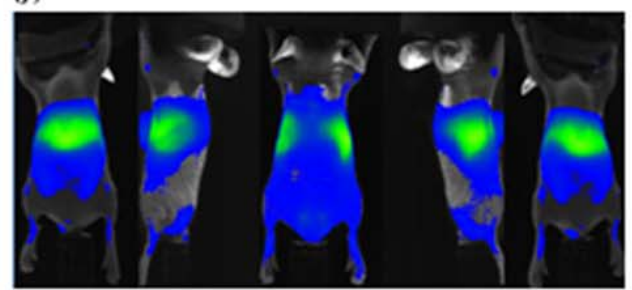

(k)

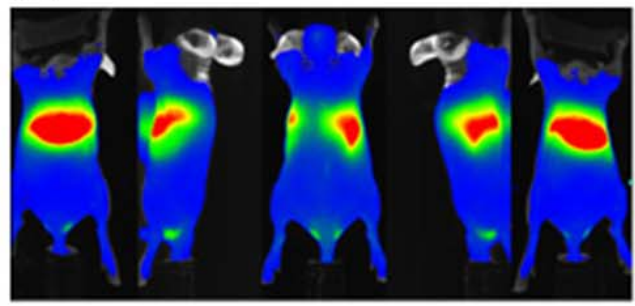

7000

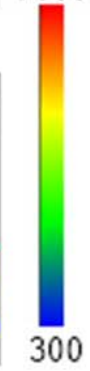


Figure 3. Pharmacokinetic change of Tri-Lacto and Mono-Lacto after the multiple administrations.

(a) Time schedule for the NIRF imaging. Black arrows show the injection time points of the Lactosomes. NIRF imagings were carried out at the times indicated by red arrows, and the alphabets below the time represent the corresponding images: after 5 min of (b) Tri-Lacto-ICG-Lacto injection

(c) Tri-Lacto-ICG-Tri-Lacto (T-T), (d) Mono-Lacto-ICG-Lacto (M-L), (e) Mono-LactoICG-Mono-Lacto (M-M) and (f) Lacto-ICG-Lacto (L-L); and after 24 h of (g) T-L, (h) T-T, (i) M-L, (j) M-M, and (k) L-L. The dotted circle indicates the position of liver.



Figure 4. The ratio of ROI at liver against background. Blue, T-L; green, T-T; red, M-L; black M-M; orange, L-L. Abbreviations are the same as those in the Figure 3 caption. The p-value (time point is 8 h): $* \mathrm{p}<0.05, * * \mathrm{p}<0.01$.

In order to analyze antigenicity of the nanocarriers, the sera obtained at day 7 after the second 
administration were subjected to ELISA (Fig. 5). Figure 5b shows the IgM amounts in sera bound to the ELISA plates respectively coated with Lacto (blue), Mono-Lacto (green), and Tri-Lacto (orange). The ELISA test using the plate coated with Lacto, which indicates the anti-poly(sarcosine) $\operatorname{IgM}$ production, clearly shows a significant reduction in the $\operatorname{IgM}$ production in the cases of Mono-Lacto/ICG-Lacto, Mono-Lacto/ICG-Mono-Lacto, Tri-Lacto/ICG- Lacto, and Tri-Lacto/ ICG-Tri-Lacto compared with that in the serum taken at day 7 after the first Lacto administration. The Sia decorated nanocarriers at the first administration are therefore effective in suppression of the immune response in the examined cases. The ELISA tests using the plates coated with Mono-Lacto and Tri-Lacto, which suggest the polyclonal IgM productions including anti-Sia IgM, show augmentation in the IgM production by the Mono-Lacto or Tri-Lacto administration. Two-time administrations of Mono-Lacto/ICG-Mono-Lacto and Tri-Lacto/ICG-Tri-Lacto further increased the IgM productions. It is speculated that the increases of the IgM production may be explained by the anti-Sia IgM production, which was also enhanced by the multiple administrations of Mono-Lacto/ICG-Mono-Lacto and Tri-Lacto/ICG-Tri-Lacto.

The antigenic determinants of Mono-Lacto and Tri-Lacto were analyzed by the competitive inhibition assay in the ELISA tests for the Mono-Lacto/ICG-Mono-Lacto serum and the Tri-Lacto/ICG-Tri-Lacto serum. Inhibitors employed here were poly(sarcosine) ${ }_{70}\left(\mathrm{PSar}_{70}\right), \mathbf{1 7}$ and $\mathbf{4}$ (Fig. $5 \mathrm{c}-\mathrm{d}$ ). PSar 70 strongly inhibited the binding of the IgM, indicating that the major fraction of IgM recognizes specifically the poly(sarcosine) block of nanocarriers. In contrast, the ELISA test of the Mono-Lacto/ICG-Mono-Lacto serum upon using the plate coated with Mono-Lacto shows no inhibitory effect neither of $\mathbf{1 7}$ nor $\mathbf{4}$, suggesting that IgM specific to the monosaccharidic Neu5Ac moiety could not be generated. On the other hand, the ELISA test of the Tri-Lacto/ICG-Tri-Lacto serum upon using the plate coated with Tri-Lacto shows dose-dependent weak inhibitions by $\mathbf{1 7}$ and 4, indicating the production of the IgM against the Neu5Ac $\alpha(2 \rightarrow 6)$ Gal $\beta(1 \rightarrow 4)$ GlcNAc $\beta$ moiety even though the fraction should be small. In addition to these saccharidic epitopes, the methyltriazine moieties in the linker between the saccharide and polymer may be recognized by some $\operatorname{IgM}$ in the 
Mono-Lacto/ICG-Mono-Lacto serum, because the augmented fraction of IgM in the Mono-Lacto/ICG-Mono-Lacto serum on the basis of the ELISA tests upon using the plate coated with Mono-Lacto from the plate coated with Lacto (Fig. 5b), which did not have any inhibitory effect of 17 and 4 (Fig. 5c), should be attributed to the other part than the saccharide and polymer moieties (Fig. 6). Similar situation has been also reported. ${ }^{34}$ 

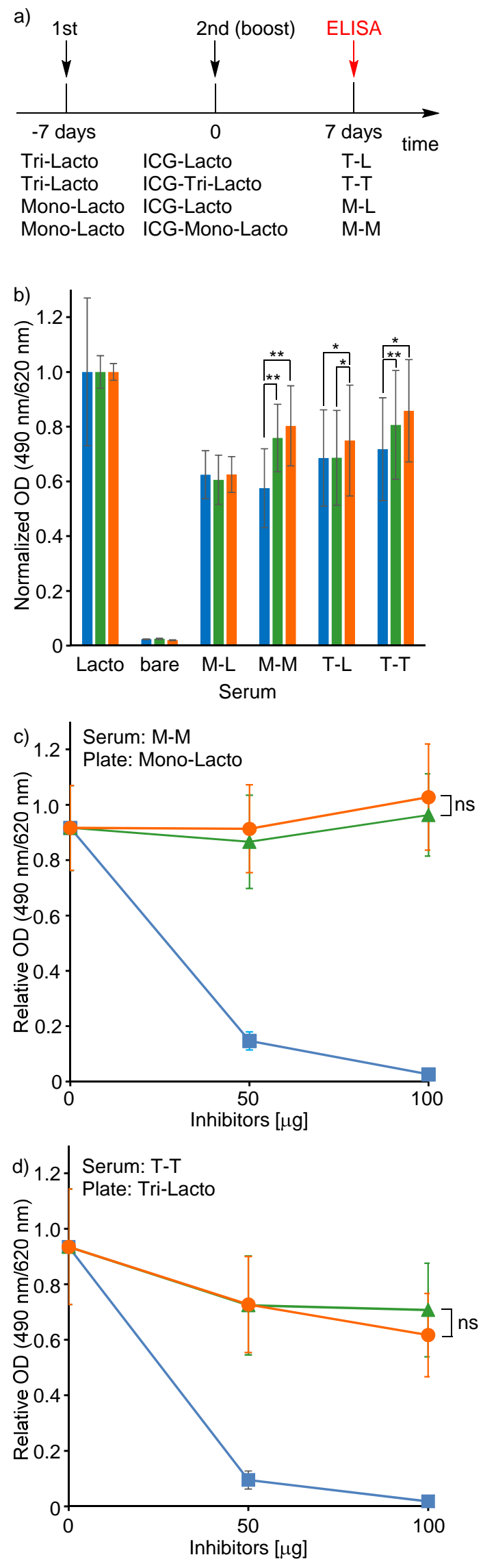
Figure 5. Specificities of the antibodies induced by multiple injection of the nanocarriers: (a) Time schedule for the ELISA analysis, T-L boosting anti-PSar immunity, T-T boosting anti-trisaccharide + anti-PSar immunity, M-L boosting anti-PSar immunity, and M-M boosting anti-monosaccharide + anti-PSar immunity; (b) amount of the IgM bound to Lacto (blue), Mono-Lacto (green), and Tri-Lacto (orange) coated plates. The data was normalized against the values for Lacto; (c) amount of the IgM in the M-M serum bound to Mono-Lacto coated plate with PSar (blue), 17 (green), and 4 (orange) as the inhibitors, (d) amount of the IgM in the T-T serum bound to Tri-Lacto coated plate with the inhibitors above. Abbreviations are the same as those in the Figure 3 caption. The p-value: not significant (ns), $* \mathrm{p}<0.05, * * \mathrm{p}<0.01$

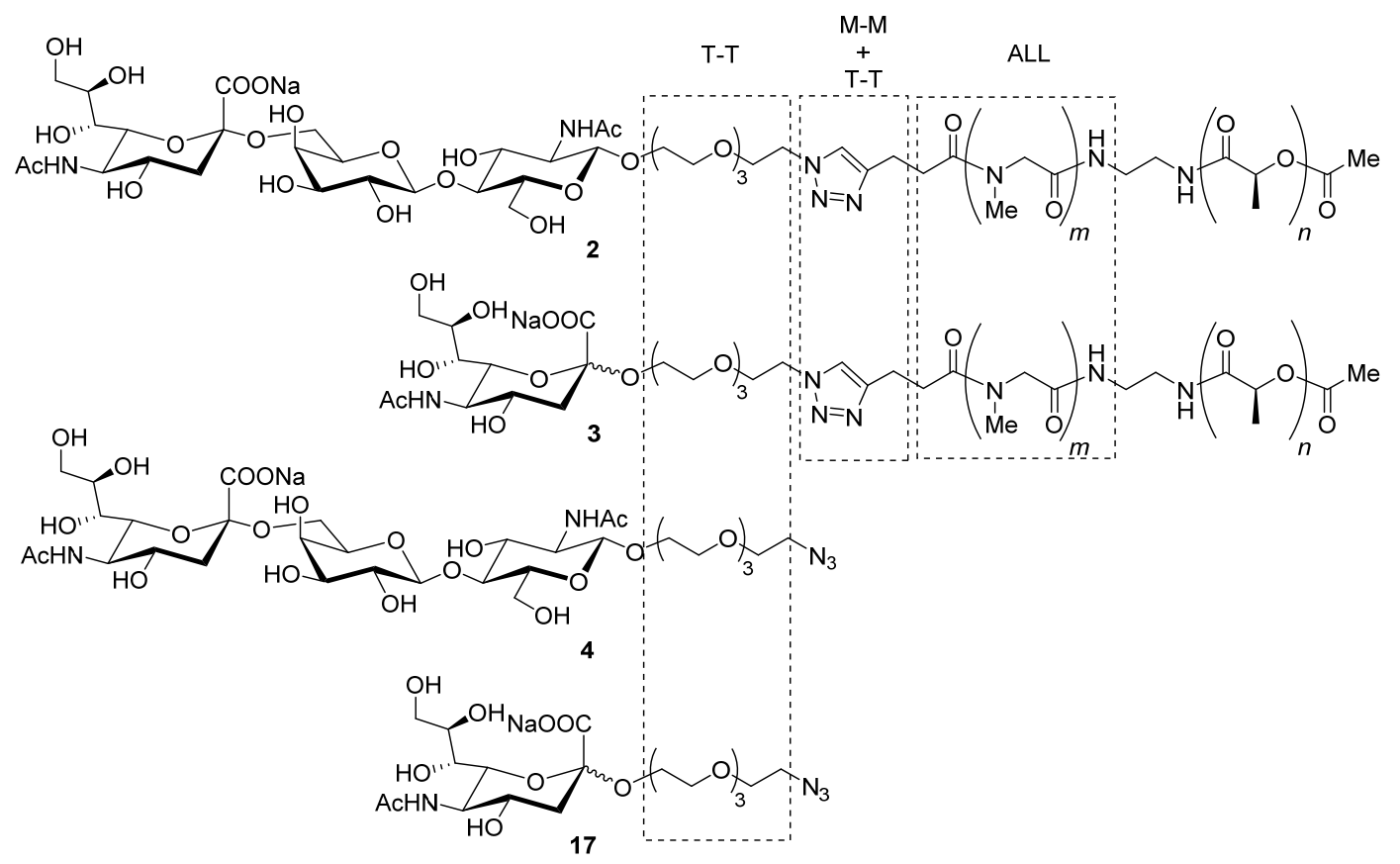

Figure 6. Predicted epitope structures for the IgMs generated in the experiments described in Figure 5. Enclosed structures by dashed lines will be recognized by the IgMs in the sera shown on the top.

It is very intriguing that the case of Mono-Lacto/ICG-Mono-Lacto showed the reduction of the liver uptake in contrast with the high liver uptake in the case of Mono-lacto/ICG-Lacto (Fig. 4). This implies that ICG-Mono-Lacto might be able to reduce phagocytosis of the opsonized nanocarriers probably by interaction of the Neu5Ac moiety with Siglec-E on immune cells, ${ }^{35}$ a member of 
CD33-related Siglecs that transmit inhibitory signals into immune cells, in addition to the conventional stealth effect of Sia coatings, ${ }^{28,29}$ which is considered to be due to the activation of the suppressive complement factor $\mathrm{H}$ through interaction with Sia ligands. ${ }^{36,37}$ The case of Tri-Lacto/ICG-Tri-Lacto also showed the limited liver uptake (Fig. 4), and the same consideration may be applied due to the specific interaction of the Neu5Ac $\alpha(2 \rightarrow 6) \operatorname{Gal} \beta(1 \rightarrow 4)$ GlcNAc $\beta$ moiety with Siglec-E. However, the case of Tri-Lacto/ICG-Lacto also showed the limited liver uptake (Fig. 4), which suggests the suppressive ABC phenomenon, could be interpreted by the reduced IgM production upon the first Tri-Lacto administration.

In conclusion, two kinds of the novel Lactosomes presenting Sia have been successfully prepared in this study. The Sia coating was effective to reduce the $\mathrm{ABC}$ phenomenon induced by the intact Lactosome due the reduction of the anti-poly(sarcosine) IgM production. The Neu5Ac $\alpha(2 \rightarrow 6)$ Gal $\beta(1 \rightarrow 4)$ GlcNAc $\beta$ coating showed the suppressive immune response better than the Neu5Ac coating reasonably due to the higher affinity with Siglec-G and CD22 to induce B-cell apoptosis. ${ }^{38}$ Further, the Sia moieties could interact with Siglec-E to suppress phagocytosis of the opsonized nanocarriers. The present study provided a novel insight into the strategy towards the molecular designs for the stealth nanocarriers, which can display both types of immunosuppressive abilities, i.e., B cell apoptosis by Siglec-G and/or CD22 and reduced phagocytic activity by Siglec-E. Now we are planning to synthesize more effective stealth nanocarriers having higher affinity against these Siglecs to enhance their abilities.

\section{Acknowledgement}

This research is partially supported by the S innovation program from Japan Science Technology Agency (JST), and Japan Agency for Medical Research and Development (AMED).

\section{References and notes}


1. Farokhzad, O. C.; Langer, R. ACS Nano 2009, 3, 16.

2. Joshi, M. D.; Muller, R. H. Eur. J. Pharm. Biopharm. 2009, 71, 161.

3. Peer, D.; Karp, J. M.; Hong, S.; Farokhzad, O. C.; Margalit, R.; Langer, R. Nat. Nanotechnol. 2007, 2, 751 .

4. Matsumura, Y.; Maeda, H. Cancer Res. 1986, 46, 6387.

5. Gref, R.; Minamitake, Y.; Peracchia, M. T.; Trubetskoy, V.; Torchilin, V.; Langer, R. Science 1994, 263, 1600.

6. Chytil, P.; Etrych, T.; Koňák, Č.; Š́rová, M.; Mrkvan, T.; Ř́hová, B.; Ulbrich, K. J. Contr. Release 2006, 115, 26.

7. Romberg, B.; Oussoren, C.; Snel, C. J.; Hennink, W. E.; Storm, G. Pharm. Res.2007, 24, 2394

8. Sheng, Y.; Liu, C.; Yuan, Y.; Tao, X.; Yang, F.; Shan, X.; Zhou, H.; Xu, F. Biomaterials 2009, 30, 2340.

9. Dams, E. T. M.; Laverman, P.; Oyen, W. J. G.; Storm, G.; Scherphof, G. L.; Van der Meer, J. W. M.; Corstens, F. H. M.; Boerman, O. C. J. Pharmacol. Exp. Ther. 2000, 292, 1071.

10. Ishida, T.; Maeda, R.; Ichihara, M.; Irimura, K.; Kiwada, H. J. Contr. Release 2003, 88, 35.

11. Mond, J. J.; Lees, A.; Snapper, C. M. Annu. Rev. Immunol. 1995, 13, 655.

12. Ishida, T.; Ichihara, M.; Wang, X.; Kiwada, H. J. Contr. Release 2006, 115, 243.

13. Ishida, T.; Ichihara, M.; Wang, X.; Yamamotko, K.; Kimura, J.; Majima, E.; Kiwada, H. J. Contr. Release 2006, 112, 15.

14. Abu Lila, A. S.; Kiwada, H.; Ishida, T. J. Contr. Release 2013, 172, 38.

15. Makino, A.; Yamahara, R.; Kimura, S. Chem. Lett. 2007, 36, 1220.

16. Makino, A.; Kizaka-Kondoh, S.; Yamahara, R.; Hara, I.; Kanzaki, T.; Ozeki, E.; Hiraoka, M.; Kimura, S. Biomaterials 2009, 30, 5156.

17. Hara, E.; Makino, A.; Kurihara, K.; Ueda, M.; Hara, I.; Kawabe, T.; Yamamoto, F.; Ozeki, E.; Togashi, K.; Kimura, S. J. Nanopart. Res. 2013, 15, 2131.

18. Hara, E.; Makino, A.; Kurihara, K.; Yamamoto, F.; Ozeki, E.; Kimura, S. Int. Immunopharmacol. 
2012, 14, 261.

19. Hara, E.; Makino, A.; Kurihara, K.; Sugai, M.; Shimizu, A.; Hara, I.; Ozeki, E.; Kimura, S. Biochim. Biophys. Acta, 2013, 1830, 4046.

20. Kim, C. J.; Hara, E.; Shimizu, A.; Sugai, M.; Kimura, S. J. Pharm. Sci. 2015, 104, 1839.

21. Mond, J. J.; Lees, A.; Snapper, C. M. Annu. Rev. Immunol. 1995, 13, 655.

22. Hara, E.; Ueda, M.; Makino, A.; Hara, I.; Ozeki, E.; Kimura, S. ACS Med. Chem. Lett. 2014, 5, 873.

23. Hara, E.; Ueda, M.; Kim, C. J.; Makino, A.; Hara, I.; Ozeki, E.; Kimura, S. J. Pept. Sci. 2014, 20 , 570.

24. Macauley, M. S.; Crocker, P. R.; Paulson, J. C. Nat. Rev. Immunol. 2014, 14, 653.

25. Duong, B. H.; Tian, H.; Ota, T.; Completo, G.; Han, S.; Vela, J. L.; Ota, M.; Kubitz, M.; Bovin, N.; Paulson, J. C.; Nemazee, D. J. Exp. Med. 2010, 207, 173, 445.

26. Macauley, M. S.; Pfrenge, F.; Rademacher, C.; Nycholat, C. M.; Gale, A. J.; von Drygalaski, A.; Paulson, J. C. J. Clin. Invest. 2013, doi: 10.1172/JCI69187.

27. Pfrengle, F.; Macauley, M. S.; Kawasaki, N.; Paulson, J. C. J. Immunol. 2013, 191, 1724.

28. Yamaguchi, H.; Yano, T.; Kato, T.; Tanaka, I.; Nakabayashi, S.; Higashi, K.; Miyoshi, S.; Yamada, H. Int. J. Pharm. 1995, 113, 141.

29. Ohyanagi, T.; Nagahori, N.; Shimawaki, K.; Hinou, H.; Yamashita, T.; Sasaki, A.; Jin, T.; Iwanaga, T.; Kinjo, M.; Nishimura, S.-I. J. Am. Chem. Soc. 2011, 133, 12507.

30. Marra, A.; Sinaÿ, P. Carbohydr. Res. 1989, 187, 35.

31. Ohmae, M.; Takada, J.; Murakami, H.; Kimura, S. Chem. Lett. 2011, 40, 438.

32. El-Faham, A.; Subirós-Funosas, R.; Prohens, R.; Albericio, F. Chem. Eur. J. 2009, 15, 9404.

33. Makino, A.; Hara, E.; Hara, I.; Yamahara, R.; Kurihara, K.; Ozeki, E.; Yamamoto, F.; Kimura, S. J. Contr. Release 2012, 161, 821.

34. Ni, J; Song, H.; Wang, Y.; Stamatos, N. M.; Wang, L.-X. Bioconjugate Chem. 2006, 17, 493.

35. Zang, J. Q.; Biedermann, B.; Nitschke, L.; Crocker, P. R. Eur. J. Immunol. 2004, 34, 1175. 
36. Pangburn, M. K. Immunopharmacology 2000, 49, 149.

37. Blaum, B. S.; Hannan, J. P.; Herbert, A. P.; Kavanagh, D.; Uhrín, D.; Stehle, T. Nat. Chem. Biol. 2015, 11,77 .

38. Paulson, J. C.; Macauley, M. S.; Kawasaki, N. Ann. N.Y. Acad. Sci. 2012, 1253, 37. 